tend to diminish the cross-section for large momentum transfers, it may be expected that it would lead to a better agreement with observation.

L. ROSENFELD.

Instituut voor theoretische Natuurkunde,

Rijks-Universiteit, Utrecht.

$$
\text { May } 5 .
$$

${ }^{1}$ Heisenberg, W., Leip. Ber., 89, 369 (1937).

2 Bagge, E., Ann. Phys., 35, 118 (1939).

' Ortner, G., Wiener Ber., 149, 259 (1940).

- Williams, E. J., Nature, 142, 431 (1938).

\title{
A New Interference Effect
}

IN the course of a class experiment demonstrating the circular Haidinger fringes produced by monochromatic illumination of a thin film between silvered surfaces, it was noticed that coloured circular fringes could be seen if white light illumination was used. The effect is best seen by looking through the film (for example, a thin air film between silvered glass plates, or a thin mica film silvered on both sides) with the eye accommodated for infinity, at a strong point source such as a 'Pointolite'. For normal illumination, the point source is seen surrounded by several rainbow fringes, as in Fig. 1, while for oblique illumination, a sharp circular white-light fringe is seen passing through the source, with concentric rainbows inside and outside, as in Fig. 2. Unlike the usual monochromatic fringes, these fringes are not influenced by lack of accurate parallelism of the silvered surfaces. The sharpness of the white-light fringe improves with increase of the reflexion coefficient of the surfaces; with plates heavily silvered by the Tolansky technique ${ }^{1}$, the fringe width is limited only by the size of the source.

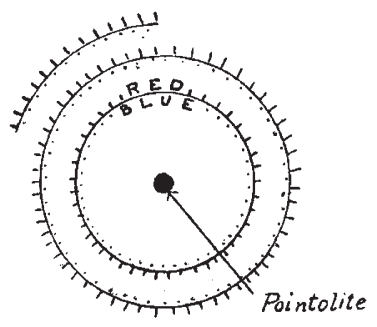

Fig. 1.

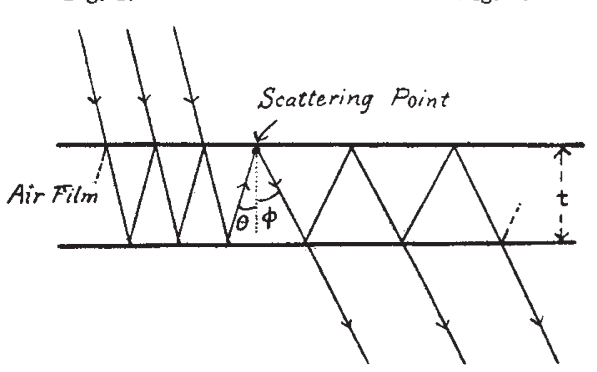

Fig. 3.

A similar effect in reflexion instead of transmission was first described by Newton and explained in detail by Stokes, who mentions that he tried unsuccessfully to observe the present effect in light transmitted through an unsilvered glass plate. We suggest the following explanation, similar essentially to that of Stokes. The fact that any illumination at all is visible off the line from the eye to the point source indicates that light is being scattered or diffracted from small irregularities in the silvered surfaces, and we suppose the fringes to be formed by light which has been multiply reflected at angle $\theta$, then scattered, and again multiply reflected at angle $\varphi$ (see Fig. 3). In the first series of multiple reflexions, light of wave-lengths for which

$$
2 t \cos \theta=m \lambda \text { ( } m \text { integral) }
$$

is strongly enhanced in transmission; while after scattering, light of wave-lengths for which

$$
2 t \cos \varphi=n \lambda \quad(n \text { integral }) \text {. . }
$$

is further strongly enhanced. If both (1) and (2) are simultaneously satisfied, then we have

$$
2 t(\cos \theta-\cos \varphi)=(m-n) \lambda \text {. . . }
$$

Thus for a given value of $\theta$ and for $m=n$, all wavelengths for which ( 1 ) is satisfied will appear strongly for $\theta=\varphi$, producing the white fringe. For $|m-n|=$ 1, 2, etc., these same wave-lengths will appear at slightly varying angles $\varphi$ given by (3), producing the various rainbows (each will appear to be practically continuous if $m$ is fairly large). The mechanism is, in fact, similar to that of the production of white. light fringes by light passed through two thin films of slightly different thicknesses (sometimes called Brewster fringes), except that in our case the second path difference is introduced in the same film after scattering.

It will be seen that the formula accounts qualitatively for the facts described, and measurements of fringe angular diameters, using an air film about $40 \lambda \mathrm{Na}$ thick, have confirmed it quantitatively. Accurate parallelism is unnecessary since for $\theta=\varphi$, $(m-n)=0$ for all values of $t$. Some further observations confirm this explanation: (a) If the silvered surfaces are accurately parallel and the 'Pointolite' is viewed against a background illuminated by sodium light, the usual Haidinger sodium fringes may be seen superimposed on the white-light fringes. It is then found that for certain values of $\theta$ (those satisfying (1) with $\lambda=\lambda_{\mathrm{Na}}$ ) the system of sodium fringes coincides exactly with the white-light system. (b) If the 'Pointolite' is replaced by a point monochromatic source (for example, a small aperture in front of a sodium vapour lamp, with precautions to avoid all stray light), fringes are seen to appear in the usual positions of Haidinger fringes whenever $\theta$ satisfies (1) but are scarcely visible at other angles. (c) Close examination of the white fringe shows that it is made up mainly of bright spots; this supports the idea that scattering is from discrete irregularities in the silvered surfaces. It is perhaps significant that the silvered plates which best showed the effect had been exposed to Manchester air for some days, which may have increased the number of scattering centres; this point, however, requires further investigation.

A more detailed treatment enables calculation of the intensity distribution in the white-light fringe, and explains the sharpening of the fringe with increase of the reflexion coefficient of the silvered surfaces, just as in the case of monochromatic Haidinger fringes.

\section{F. K. BaUChwitz}

Cavendish Laboratory,

D. SHOENBerg.

Cambridge.

June 1.

1 Tolansky, "High Résolution Spectroscopy" (Methuen), in the press. "Stokes, Trans. Camb. Phil. Soc., 9, 147 (1851) ("Collected Papers", $3,155)$. 